\title{
SOBRE LA INTRODUCCIÓN DEL PRINCIPIO DE DUALIDAD EN GEOMETRIA PROYECTIVA
}

Mario H. Otero

INSTITUTO DE INVESTicaciones FLosóficas Universidad Nacional Autónoma de México

1. En el momento en que Félix Klein presenta su Programa de Erlangen, la geometría proyectiva está plenamente desarrollada. Al aplicar a ella la teoría de los grupos debida a E. Galois, la matemática moderna está ya constituida; aunque no ha alcanzado el nivel, por ejemplo, de Bourbaki, sin embargo trata de las "mismas cosas" que la matemática de nuestros días. Los números y figuras no son ya su objeto, las estructuras abstractas son su tema de ocupación. Muchas etapas quedaban por recorrer cuyos hitos, salvo uno, no voy a señalar aqui.

En 1899, D. Hilbert publica sus Fundamentos de la geometria, ${ }^{1}$ donde los Elementos euclídeos aparecen bajo nueva forma. Bajo una común estructura -a menos de detalles- Hilbert y Euclides reconstruyen la geometria llamada elemental. Pero, tras una engañosa apariencia, se deslindan dos disciplinas muy diferentes: una geometría patentemente fisica $^{2}$ en el caso de Euclides y una geometría "euclídea" no física en el caso de los Grundlagen. Trazar la historia de la aparición de la matemática abstracta no es tarea fácil, aunque varios intentos incompletos ya hayan tenido lugar. De todos modos la comparación revela que no es necesario apelar a geometrias divergentes ni abstrusamente atractivas para captar el cambio acontecido. De Euclides a Hilbert existe un abismo que se puede marcar, primariamente, con sólo decir que el conjunto de nuevos axiomas apelan a tres clases cualesquiera de cosas que los satisfacen, mientras que en los Elementos sólo estaban en cuestión clases de cosas muy concretas: puntos, rectas y planos no eran antes cualesquiera, como para Hilbert, sino esencialmente entes fisicos. Cómo, dentro de una "misma" geometría, acontece un cambio de ese tipo debe ser explicado adecuadamente y esto es, como deciamos, tarea aún abierta. Su importancia no se escapa ni requiere ser especificada.

1 Grundlagen der Geometrie, Leipzig, Teubner, 1899. Traducción inglesa: Foundations of Geometry, Chicago, Open Court, 1910.

2 El concepto de geometria física ha sido usado entre otros por B. Russell. Aquil tomamos como base Wilder, R., Introduction to the Foundations of Mathematics, Nueva York, 1952. 
En lugar de tratar el desbordante conjunto de problemas vinculados a esta tarea pueden elegirse ciertos temas que aparecen como especialmente significativos. Muy probablemente una decisión parcial acerca del proceso referido pueda ser efectuada, no en bloque, o a través del análisis de complejos pasos, sino a través del tratamiento de algunos aspectos que podríamos considerar claves para su comprensión. Enfocaremos uno de ellos o, mejor dicho, un asunto de detalle aparente, que no puede considerarse siquiera como uno de esos aspectos claves en su totalidad.

La introducción del principio de dualidad, en una forma muy primitiva pero suficientemente eficaz, en los años veinte del siglo pasado, puede servir del modo indicado. No nos interesa dilucidar las extendidas polémicas acerca de la precedencia de quienes lo utilizaron, sino especificar un poco su función en el desarrollo de la geometría, comparándola con la que cumplieron efectivamente conceptos o teorías más espectaculares.

2. Ya en la geometria analítica cartesiana se da un cambio en cuanto a los "objetos" del discurso geométrico. En particular, en el caso de la geometría plana, la correspondencia entre pares de números -coordenadas- y puntos, y entre ecuaciones y rectas, con un significado geométrico preciso de los parámetros, introduce un nuevo lenguaje acerca de las relaciones entre puntos y rectas en las que ellos no aparecen para nada como tales. Un "punto" $P(a, b)$ pertenecerá a una "recta" $y=m x+$ $n$ si y sólo si $b=m a+n$. En este caso, los puntos y rectas -en rigor, al llamarlos así se estarían usando nombres arbitrarios- no poseen sólo las propiedades asumidas desde siempre sino otras propiedades nuevas (por ejemplo, que en el ejemplo referido las coordenadas sátisfacen la ecuación), si bien correspondientes a aquéllas. Así, el discurso de la geometría analítica del plano adquiere una nueva forma, y los "objetos" son otra cosa que puntos y rectas sensu stricto.

Sin embargo, la producción de esta nueva geometría se efectúa de tal modo, que la nueva versión reproduce en un lenguaje isomorfo las propiedades ya conocidas. La geometría analítica aparece como una construcción adaptada especialmente para el cálculo de posiciones y de trayectorias —elementos físicos-: nuevamente, pues, como geometria física, y en este sentido no constituye una innovación radical. Lo que interesa a Descartes es algo más que la trayectoria de una mosca en el invierno holandés; no obstante, en el nuevo cálculo se reproducen las estructuras que interesan para el control de procesos físicos como ese zumbador vuelo. La naturaleza y el alcance de las aplicaciones son esclarecedores al respecto. Por un lado, la geometría euclídea cartesiana reproduce, con objetos aparentes distintos, relaciones bien estudiadas; sólo que lo hace 
con un nuevo rendimiento teórico. Ya en este caso se presenta, en tenue esbozo - y con una ambigüedad que veremos persistir - , el proceso de transformación de la geometría euclídea. Ya aquí se produce más de lo reproducido; por ejemplo, las soluciones de un sistema de dos ecuaciones de primer grado con dos incógnitas correspondientes a los puntos de intersección de rectas en el plano (1) admiten otras interpretaciones (paralelismo o superposición) distintas a la propuesta y, aun en ella, (2) el estudio de los parámetros aporta una generalidad insólita. De ahí el interés de este "primer" tímido paso en la larga marcha hacia la geometría abstracta.

3. El estudio de las cónicas tiene una historia de veintitrés siglos. Sin embargo, durante doce de ellos, desde Papus hasta el comienzo del dieciséis, no experimentó progresos. Coodlige nos dice que, si excluyéramos a Papus, serían diecisiete siglos de quietud. De golpe, el interés por el tema se renovó y desembocó en lo que hoy conocemos como geometría proyectiva. Desargues, Pascal y, particularmente, Poncelet, se destacan entre los nombres de quienes cumplieron este proceso.

3.1 Antes de llegar a nuestro tema específico conviene recordar algunos momentos significativos del desarrollo de la geometría proyectiva. En primer lugar, señalar que la introducción de elementos impropios --.especialmente puntos- en la geometría esférica, con anterioridad a aquel desarrollo, no se integra en él, por más que hoy pueda considerarse como un antecedente a tener en cuenta; en geometría proyectiva debió procederse nuevamente, y en forma totalmente independiente, a introducirlos. En segundo lugar, las investigaciones de Desargues sobre las cónicas permanecieron sin conocerse durante un largo periodo al punto que el Brouillon projet sobre las intersecciones de un cono con un plano, de 1639 , solamente fue localizado y estudiado recientemente y sus resultados sólo se conocieron antes muy parcialmente, a través de Pascal y La Hire. En tercer lugar que las nuevas investigaciones sobre las cónicas, las que correspondieron a la geometra proyectiva avanzada, con Poncelet, prescinden del cono para constituirse como una respuesta a un problema, por ejemplo, del plano acerca de ciertas curvas. Si bien corresponden a Desargues varios teoremas fundamentales, sólo con posterioridad aparece la disciplina como constituida sistemáticamente. Tampoco son suficientes para ello los trabajos de Pascal y La Hire. Pascal escribe, a los dieciséis años, un tratado sobre las cónicas que permanecerá inédito. Sin embargo en 1640 publica un opúsculo Essais pour les coniques ${ }^{3}$ donde presenta los principales teoremas por él descubiertos, especialmente uno central, referente a los exágonos inscritos en una cónica. La Hire introduce una teoría de polos y polares como base de su geometría. Con todo, los ele-

3 Pascal, B., Essais pour les coniques, 1640. 
mentos referidos en los autores citados son insuficientes y sólo el desarrollo de la geometra descriptiva, especialmente por G. Monge, va a fertilizarlos. Construida en ocasión de las necesidades de trazar planos de fortificaciones, la geometría descriptiva -matemáticamente poco importante en otros sentidos- provee un método de trabajo para la naciente geometría proyectiva. La obra de Monge ${ }^{4}$ y la de Carnot ${ }^{5}$ permiten clasificar las propiedades de las figuras en métricas y descriptivas, lo cual juega un rol central por más que solamente haya podido fundarse con rigor recién cuando, mucho más tarde, se hace intervenir la noción de grupo en geometría.

Es la obra de Poncelet ${ }^{6}$ que llega a constituir en disciplina sistemática la geometría proyectiva: es el Tratado sobre las propiedades proyectivas de las figuras que la presenta de modo integrado, más allá de cierto primitivismo en su conceptuación. El uso de dos operaciones - la proyección y la sección - y de la homología le permiten hacerlo. Dos principios, el de "continuidad" y el de dualidad complementan los elementos referidos. Es Poncelet quien considera al conjunto de puntos impropios del plano como recta impropia (en cambio Desargues usaba los puntos impropios con un alcance más limitado).

Con posterioridad la introducción en geometría proyectiva, por Chasles y Möbius, de la relación anarmónica conocida ya por Papus, y más tarde, de las seis formas proyectivas básicas, con la noción de proyectividad entre dos formas, abren el camino para una sistematización más amplia, y para la generación proyectiva de las cónicas. Es von Staudt quien introduce una nueva definición de las cónicas basada en la polaridad a priori. Con ello estaba dada la geometría proyectiva en su forma sistemática básica, sin perjuicio de los pasos que tuvieron lugar hasta Klein, en el periodo que nos interesa aquí. A esos pasos vamos a referirnos con posterioridad.

3.2 Pueden, en todo ello, distinguirse, hasta Klein, fundamentalmente tres periodos. 1) Un primero en el cual aparecen los elementos básicos que van a servir para constituir la disciplina y algunos teoremas básicos. En dicho periodo, como es obvio, no existe una comunidad de investigadores que posean un corpus de doctrina lo suficientemente desarrollado. 2) Un segundo periodo; a partir de Poncelet en que la geometría proyectiva está constituida y existe una comunidad de investigación -predominantemente francesa en un primer momento, luego alemana, luego

4 Monge, G. Sus obras de fundación de la geometría descriptiva, su labor docente y de organización en la École Polytechnique y su capacidad matemática abrieron paso a la geometría proyectiva naciente. Poncelet se formó con él.

5 Carnot, L., Géometrie de position, 1803.

6 Poncelet, J. V., Traité des proprietés projectives des figures, Paris, 1822. 
aún más amplia, incluyendo por ejemplo a Cayley, ${ }^{7}$ de importancia central; periodo en el que la geometría proyectiva se desarrolla sobre la base del Tratado de Poncelet y se proponen algunas modificaciones sistemáticas importantes, aunque no decisivas. Las consecuencias del principio de dualidad son explícitas al promediar el periodo y se busca fundamentarlo (por demostración, en una de las variantes). 3) En el tercer periodo no sólo la comunidad de geómetras especializados desarrollan la disciplina sino que se fertiliza la teoría con aportes de otras disciplinas matemáticas. De cualquier modo la característica común de estos dos últimos periodos es la existencia de una continuidad de trabajo. Esto toma importancia cuando se comparan los aportes en relación con otros y se atiende a la penetración de las matemáticas en sentido abstracto contemporáneo.

4. Consideremos un aspecto en esa historia, de la que dimos apenas algunos rasgos. Más allá de la introducción de elementos en el infinito -puntos y rectas impropios_, más allá de las especiales propiedades del hexágono de Pascal o de teoremas significativos en ese desarrollo, vamos a considerar por el momento sólo la dualidad en geometría proyectiva.

4.1 Las proposiciones fundamentales de esa geometria admiten el in. tercambio de las expresiones "punto" y "recta" en el plano y "punto" y "plano" en el espacio. Estas leyes de simetría conceptual se introducen en toda la geometría del plano y del espacio, respectivamente.

No vamos a considerar todas las vicisitudes que sufrió la dualidad - justamente la simetría indicada - y menos aún como dijimos las disputas por la precedencia en su introducción. La dualidad aparece en Poncelet a través de su estudio de la polaridad de las cónicas (polos y polares hacen de mediación para la dualidad) mientras que en Gergonne ${ }^{8}$ aparece como principio general que establece una relación entre teoremas (si en determinados teoremas de la geometría proyectiva plana se sustituyen las palabras "punto" y "recta" por "recta" y "punto" respectivamente, se obtienen teoremas llamados duales de los de partida, y reciprocamente). La dualidad en Poncelet queda particularizada, en Ger-

7 Cayley, A., The Collected Mathematical Papers of..., Cambridge, 1889-1897.

8 Gergonne, J. D. (ed.), Annales de Mathématiques Pures et Apliquées, Nismes, 1810-1831. Los Annales, aparte del ser la primera revista estrictamente matemática jamás publicada, fueron el lugar principal de las publicaciones de geometría proyectiva en la época fundacional. Gergonne mismo produjo numerosas contribuciones a la misma y en particular los artículos dedicados al principio de dualidad principalmente: "Recherche de quelque-unes des lois générales qui régissent les polyèdres", V.15, 1824-25, pp. 157-164, "Considérations philosophiques sur les éléments de la science de l'étendue", V. 16, 1825-26, pp. 209-231, y "Recherches sur quelques lois générales qui régissent les lignes et surfaces algébriques de tous les ordres", V. 17, 1826-27, pp. 214-252. 
gonne generalizada aunque con poco cuidado. No aparece tampoco sujeta a demostración y se la juzga en general a partir de los resultados que produce.

A medida que es aplicada se va afinando su uso aunque sin cambios en lo esencial. Möbius ${ }^{\ominus}$ se expresa como sigue:

De toda proposición en base a la cual de un sistema de puntos y rectas arbitrariamente elegidos en el plano, mediante sucesivas uniones e intersecciones, son deducidos otros puntos y rectas, de los cuales tres dados de los primeros yacen sobre una recta, o tres dadas de las segundas se encuentran en un punto, se puede deducir otra en la cual los puntos se intercambian con las rectas y 'yacen sobre una recta' con 'encontrarse en un punto'.

Las versiones que aparecen sucesivamente introducen pequeños cambios; como ejemplo incluímos en primer lugar la de von Staudt: ${ }^{10}$

Las primeras proposiciones de la geometría hacen ya sentir cierta ley de reciprocidad o de dualidad, gracias a la cual en el espacio, el punto y el plano están uno frente al otro, y toda proposición en la cual no se haga ninguna distinción entre elementos propios e impropios, encuentra su complementaria en otra que resulta de la primera cambiando entre $s$ punto y plano... Dos proposiciones de tal especie se colocan corrientemente una al costado de la otra como los dos aspectos de una única proposición.

Otra forma en que aparece la dualidad se expresa del modo siguiente:

Se puede asignar a cada sistema $\Sigma$ un sistema recíproco (dual), $\Sigma_{\perp}$. A todo par de formas $G, G^{\prime}$, colineares en $\Sigma$ corresponde un par de formas colineares entre sí en $\Sigma_{1}$. A cada elemento de $\Sigma$, unido respecto a las dos primeras formas, corresponde un elemento en $\Sigma_{1}$ que está unido respecto a las dos últimas. ${ }^{11}$

Finalmente, Clebsch, sobre la base de entender las cónicas, sea como conjunto de puntos, sea por vía de sus tangentes, nos dice: "ciertas relaciones valederas para conjuntos de puntos se pueden transportar a figuras de rectas".

9 Mobius, A. F., "Der baricentrische Calcul" (1827), parágr. 285, en: Gesammelte Werke, Leipzig, Hirzel, 1885. Citado por: Nuvoli, L., "Il principio di dualita in geometria visto attraverso le successive sur formulazioni fino agli inizzi del secolo XX" en Physis, V. 2, 1960, pp. 101-120.

10 Staudt, K. V., Geometrie der Lage, parágr. 6, n. 66, Nurberg, 1847. Citado en Nuvoli, op. cit.

11 Staudt, K. V., ibid., n. 137. 
Quedan de lado aquí los problemas vinculados a la "demostración" del principio de dualidad pero no es eso lo que interesa aquí sino $s u$ significado. Cabe solamente señalar que esa demostración recorrió las vías de la geometría proyectiva algébrica y no los caminos sintéticos tradicionales en la disciplina.

4.2 Tomando, por su relativa simplicidad, la geometría proyectiva plana, en su presentación dual, que se hace viable por la introducción de elementos en el infinito -en particular, de puntos impropios-, se desarrolla del modo siguiente. Los sistemas geométricos presentan un "paralelismo" (en esta geometría que trata las paralelas de modo tan singular, cortándolas justamente en un punto impropio), de modo que son posibles dos presentaciones duales; una de las cuales incluye puntos y rectas en los lugares donde en la otra aparecen, respectivamente, rectas y puntos, dentro de estructuras lingüístico-relacionales incambiadas.

Esta forma dual de presentar las propiedades de objetos geométricos supone un principio de dualidad cuya significación nos interesa, y llega hasta nuestros dias en las formas usuales de construir la geometría proyectiva, es decir, en las formas axiomáticas correspondientes al estado actual de nuestras concepciones deductivas.

4.3 Sin necesidad de explicitar en nueva forma tal principio, la presentación dual misma introduce ya una manera diferente de entender la geometría. Dada la referida forma de presentación dual, la geometría puede concebirse construida por una columna $\mathrm{A}$-o por otra $\mathrm{B}$-, o bien (más razonablemente), por la presentación completa que comprende las dos columnas a la vez. Se trata, como dijimos, de dos sistemas deductivos "paralelos" en los que "puntos" y "rectas" aparecen determinados del modo indicado.

Ahora bien, podemos preguntarnos de inmediato cuáles son los objetos de esta geometría y de qué modo son susceptibles de una interpretación fisica. Aunque la idea de magnitud -longitud de un segmento (en la forma de congruencia de segmentos), por ejemplo- haya sido eliminada, interesando sólo las propiedades justamente proyectivas, podemos preguntarnos si esta geometría podría interpretarse de manera que el dominio estuviese formado por elementos físicos - posiciones o trayectorias-, tal como podían interpretarse la euclídea y la analítica. Y, más aún, podemos preguntarnos qué cosas designan las expresiones "punto" y "recta" en este uso tan peculiar. Es esto precisamente lo que aparece como una novedad significativa.

Ambas columnas, lefdas alternadamente, constituyen la geometría proyectiva. Se trata de dos sistemas deductivos - no necesariamente axiomáticos en nuestro sentido- isomorfos, i. e., de igual estructura, cuyas variables toman como valores rectas y puntos, con un nuevo contenido 
respecto a los anteriores referidos. En ese caso la geometría presenta una única estructura con variables ( $\alpha$ y $\beta$ ) a sustituir adecuadamente. La geometría es esta estructura, suponiendo obviamente las reglas de sustitución de las variables. Éstas, por tanto, no poseen, como es obvio, una designación fija, es decir, valores euclídeos físicos determinados (lo cual responde, de paso, a la primera pregunta).

El salto producido frente a la geometría física euclídea es notorio, y esto constituye ya no un modo nuevo de construir la misma geometría, sino una nueva manera de concebir la geometria y sus objetos. La estructura presentada, a través de un conjunto de propiedades (axiomas y teoremas), desplaza la idea previamente básica de propiedades de los puntos y de las rectas.

De esta manera se cumple un cambio acerca de lo sustancial en la geometría. Este cambio se ha atribuido tradicionalmente a momentos posteriores y distintos al de la geometría proyectiva. Sin embargo, no cabe duda alguna que ya en ésta aparece, en la forma de presentarse los sistemas deductivos, un salto radical, y no sólo un perfeccionamiento más, o un desarrollo de formas geométricas preexistentes. Por ello, insistimos, la presentación dual expresa, hace explícito, un modo radicalmente distinto de encarar las propiedades geométricas. Con la especificidad introducida por la dualidad se trata pues de algo más que del mero descubrimiento de teoremas en una rama nueva de la geometría.

Una somera comparación con el caso de la geometría analítica antes referido muestra notorias diferencias, dentro de una línea de desarrollo que nos interesará indicar luego.

En el caso anterior el lenguaje - geométricamente- habia cambiado, pasando a llamarse 'punto' un par ordenado de números reales y 'recta' una ecuación de determinado tipo. Pero la separación entre los dos tipos de entes se mantenía, más allá del hecho de que ciertas propiedades de números y ecuaciones "reflejasen" propiedades de puntos y rectas estrictamente euclídeos. Por otra parte, la fisicidad de la geometría analítica, por lo menos en sus formas primitivas, la enlazaba con su predecesora euclídea. Por el contrario, las características señaladas de la geometría proyectiva hacen ya de ella una geometría "no-euclídea", aunque en un sentido muy especial de la palabra. Teniendo en cuenta la posición de Frege ${ }^{12}$ frente a la concepción hilbertiana de la geometría "euclídea", no es inútil señalar el paso nada desdeñable ya cumplido por la geometría proyectiva en la concepción misma de la geometría, y aun de la matemática y de los sistemas deductivos en general. Ese paso es sólo uno de los posibles, y de los efectivamente realizados más tarde, en ese sen-

12 La polémica sostenida entre Frege y Hilbert es especialmente iluminadora a este respecto. 
tido. Sólo que posee, por el momento en que fue dado, una significación histórica particularmente señalable. Por ello, había que señalar este hito. Dejarlo de lado, pasando en forma excesivamente rápida y ligera a considerar otros momentos, sería desconocer una verdadera revolución científica; error indudable, a nuestro modo de ver, pero casi invariable en la literatura.

5. Si el principio de dualidad tiene los efectos referidos, el desarrollo global de la geometría proyectiva posee nuevos caracteres propios que es necesario señalar antes de dar un paso más.

No basta con los aspectos reseñados o aludidos en 3.1. Se trataba alli de algunos de los elementos que constituyeron a la geometría proyectiva como disciplina autónoma y distinta a las anteriormente existentes. Frente al auge avasallador de la geometría analítica se vivió una reacción de la geometría sintética que parecía de algún modo agotada en ese momento y restríngida a procedimientos de corto alcance comparados con los analíticos. Sin embargo, no sólo el principłs de dualidad sino algunos métodos introducdos en geometría permitierron una "cosecha" enorme de teoremas y especialmente ciertos encadenamientos especiales que dieron perfil a la disciplina. Eso no quiere decir que no existieran gentes como Gergonne o como Plücker que usaran métodos analíticos para tratar "la materia" de la geometría proyectiva. Ello fue fructífero dentro de ésta. Tampoco quiere decir solamente que gentes como Steiner no rechazaran vivamente esos métodos analíticos reivindicando una geometría pura, estrictamente sintética. Quiere decir más bien que con unos u otros procedimientos se tendió a constituir una disciplina diferente a la geometría analítica por su "objeto" y por sus métodos. En ello no vamos a entrar aquí.

Pero hemos de señalar un rasgo importante. La geometría proyectiva original pretende ser una geometría no métrica, una ciencia de las propiedades proyectivas de las figuras. Sin embargo, el desprendimiento de las nociones de congruencia no se efectuó en un principio de manera radical y por el contrario las relaciones anarmónicas suponían definiciones en base a congruencia que luego se "reabsorbían" en el resto de la construcción. Las tendencias puristas a que hemos hecho referencia, la tendencia a una geometría estrictamente sintética, llevaron a von Staudt a definir geométricamente -con elementos exclusivamente proyectivos- dichas relaciones anarmónicas. Ya no se establecieron como relaciones de proporcionalidad entre segmentos medidos - la medida debía ser excluída- sino que von Staudt generó un modo de construcción exclusivamente no-métrico para definir aquellas relaciones. La tendencia purista lleva asimismo a este autor a la construcción de un cuerpo "axiomático" para la geometría proyectiva en un momento en 
que las axiomáticas eran inexistentes en sentido moderno. A tal punto la geometría proyectiva exigía una construcción de ese tipo, por los problemas que planteaba - y que luego plantearon agudamente las geometrías no-euclidianas (por ej. consistencia)—, que M. Pasch, ${ }^{13}$ autor de la "primera" axiomática moderna, la construye a los efectos de presentar justamente a la geometría proyectiva de un modo adecuado. La construcción correspondiente para la geometría euclídea - debida a Hilbert (1899) - resulta un paso muy posterior frente a los intentos de von Staudt para la geometría proyectiva y al sistema axiomático de M. Pasch para esta misma rama de las matemáticas.

El contenido nuevo de la geometría proyectiva (3.1) se vio complementado pues por una tendencia al rigor, a la presentación axiomática, que luego permearía el total de las matemáticas y que ya se iba insinuando también en otras de sus ramas.

Conjuntamente habría que considerar la obra de Cayley, culminada luego por S. Lie y por Klêt, en un sentido ya indicado; el tema, muy significativo para la matemática moderna, y producido justamente a partir de la geometría proyectiva llevó a la idea de geometría abstracta, de geometrías equivalentes y de geometrías subordinadas, pero ésta es, aunque muy vinculada, otra historia. El hecho esencial a retener es la función cumplida por la geometría proyectiva en este proceso (ello se refleja además sin duda en el tratamiento posterior de Hilbert para la geometría euclídea y conexas).

5.1 Los dos aspectos indicados en 3.1 y 5 de la geometría proyectiva, es decir, construcción efectiva y pasos hacia un rigor entonces inusual, muestran - por más que serían necesarios algunos elementos históricos más para probarlo acabadamente- que en la geometría proyectiva existe desde los años veinte del siglo xix, en forma continuada, una comunidad de investigación, una tradición científica, un conjunto de problemas y un desarrollo tales que la configuran como una disciplina en marcha. A los efectos comparativos etso basta (cf. 6).

Hasta el momento, hemos insistido en tres puntos, el sentido novísimo del principio de dualidad, los elementos que hacen de la geometría proyectiva en su conjunto un sistema especialmente interesante en la transformación de las matemáticas en el siglo xIx, y el carácter progresivamente constructivo de los resultados del trabajo de una comunidad científica integrada y especialmente activa.

Veamos ahora cómo comparar ese desarrollo con otros en gran medida convergentes.

13 Torretti, R., Philosophy of Geometry from Riemann to Poincaré, Dordrecht, Reidel, 1978. 
6. La geometría proyectiva y las geometrías no euclidianas ${ }^{14}$ tuvieron un desarrollo independiente entre sí en un primer, largo periodo. En 5.1 señalamos la continuidad de trabajo que se dio en la primera. En cambio las geometrías no-euclídeas tuvieron un proceso de difusión totalmente distinto. No hubo en sus comienzos una comunidad de investigación integrada, por más que los trabajos de Bolyai y Lobatchevsky ya constituían presentaciones de algún modo acabadas de sus temas. Los trabajos de esos autores son de 1832 y 1829 respectivamente, el de Riemann de $1854^{15}$ y el de Beltrami de 1868. Debieron pasar cuarenta años prácticamente para que se trabajara coordinadamente en geometrías noeuclideas y para que se gestara una comunidad de investigación. No entro en los detalles, que aparecen bien estudiados en la literatura.

Es decir que, más allá de los contenidos a que hemos hecho referencia, la constitución de comunidades de investigación en los dos casos es totalmente distinta. Cuando la geometría proyectiva poseía signos inconfundibles de madurez, de integración con otras ramas de la matemática y de efectos sobre el conjunto de la investigación matemática, las geometrías no-euclídeas estaban todavía sujetas a un difícil proceso de difusión y para nada integradas del mismo modo.

Por ello puede afirmarse que los efectos matemáticos de uno y otro campo de estudio tenían que ser muy distintos. $\mathrm{Y}$ hasta podría decirse, por vía de hipótesis, que, además, lo esencial del aporte de la geometria proyectiva fue oscurecido por los efectos filosóficos de las geometrias noeuclideas -efectos positivos y negativos de resistencias__ en momentos posteriores, momentos que otros factores adicionales hicieron propicios para ello.

7. Frente a la opinión corrientemente sostenida de que la gran transformación de la geometría en el siglo xIx tuvo lugar con la introducción de las geometrías no-euclidianas y sólo secundariamente en relación con otros desarrollos, y que la geometría en su sentido abstracto se produjo en base a aquéllas, se sostiene pues en el presente trabajo que la geometría proyectiva cumplió un papel específico importante y no reconocido. En primer lugar,

1) Las geometrías no-eucliclianas se desarrollan diferidamente a partir de Lobatchevski y de Bolyai - a su vez separados entre si- no contando, para la generación de la tradición científica, los vislumbres que tuvieron

14 Para la interpretación supuesta acerca de las geometrías no euclídeas nos remitimos a un trabajo anterior, "Algunas observaciones acerca del debilitamiento de la geometría física", Diánoia, 1980, del que por otra parte se han retomado antes algunos trozos que son necesarios para la comprensión del resto.

15 Aunque el trabajo de Riemann es de esta fecha fue publicado recién en 1867; por otra parte la conferencia fue dada originalmente ante un grupo muy reducido de auditores. 
lugar con anterioridad, por más que hoy ellos puedan ser considerados como antecedentes legitimos.

2) Las geometrías así introducidas no se constituyen inmediatamente en campo de trabajo ni en tema de tradición científica con una elaboración más o menos continuada. Por el contrario han de transcurrir nada menos que cuatro decenios antes de que tal tradición se constituya, de modo que los trabajos tanto de Bolyai como de Lobatchevski, como alguno fundamental de los posteriores de Riemann, aparecen como hitos aislados. La falta de difusión -debida muy probablemente al carácter marginal de los centros universitarios en que se producen- Budapest y Kazan respectivamente, para los primeros, se constituye en factor central que determina el carácter aislado de los descubrimientos y la falta de consecuencias inmediatas de los mismos.

En segundo lugar,

3) Aunque la geometría proyectiva se constituya como disciplina autónoma en los mismos años veinte que las geometrfas no euclidianas, los trabajos anteriores - aún con las anomalías en la difusión de la obra de Desargues — son una base importante para el tratado de Poncelet $y$, de cualquier modo, éste no se sitúa como obra aislada sino que es inmediatamente seguido por un trabajo normal en la disciplina. Hemos referido tanto para la geometría proyectiva misma —en que se sitúa el carácter disciplinario-, como para el principio de dualidad - que constituye un ejemplo de los aportes donde se sitúa la novedad respecto a las geometrías no-físicas-, algunos elementos históricos. Ellos nos permiten afirmar esa continuidad de trabajo de una comunidad científica especializada. Permiten asimismo — sin necesidad de repetir una historia completa de la disciplina - ver cuáles son los aportes dentro de esa continuidad de trabajo.

4) Hemos distinguido tres periodos en la historia de la geometría proyectiva anterior a Klein, dos de los cuales _los últimos-- poseen características diversas pero sin solución de continuidad. Uno en que se trabaja en los temas mismos de la geometría proyectiva - sea en su versión sintética como en sus versiones algébricas o "mixtas" - y otro en que, además, se introducen en geometría proyectiva elementos de otras disciplinas matemáticas. Son dos formas de trabajo en que se expresa, como dijimos, una misma continuidad y unificación de intereses y temas.

En tercer lugar,

5) El cambio fundamental que se produce en la geometría, a través de la geometría proyectiva, y en especial a través del principio de dualidad, agregado a la continuidad referida y a la constitución temprana de una comunidad de investigación, hace que podamos afirmar - sin excluir el aporte para nada indudable de las geometrías no-euclidianas- 
que la geometría proyectiva por sí misma produce un momento, o quizás un periodo, de giro en la manera de hacer geometría y en la concepción misma de ésta que debe ser reconocido en toda su amplitud y en sus consecuencias, comparativamente tempranas. En este sentido queremos contribuir a fechar o a acotar fundadamente el momento en que se produce la transformación hacia las matemáticas modernas, tema de indudable importancia y sobre el que apenas se ha dicho algo, permaneciendo situado en un periodo inadecuadamente demasiado prolongado. En Hilbert el cambio está concluido y asumido, ¿dónde se podría situar con una indeterminación algo menor en las cotas? 